\title{
ONTARIO MATHEMATICAL MEETINGS
}

The second Ontario Mathematical Meeting was held Saturday, December 10, 1966 at Sidney Smith Building, University of Toronto. Research papers were presented in the morning, followed by lunch at Massey College, followed by a one-hour address by Professor S. Sakai (University of Pennsylvania) entitled: Derivations of $W^{*}$-algebras. Abstracts of research papers which were presented are as follows:

66.7 N.M. dos Santos (Queen's University) On the Local Distribution of Singularities of Differentiable Maps

Let $\varphi: M \rightarrow L$ be a differentiable map of manifolds, $M_{m}^{k}$ the space of $k$-th order tangent vectors at $m \varepsilon M$, and $\mathrm{s}^{\mathrm{k}}\left(\mathrm{M}_{\mathrm{m}}\right)$ the symmetric $\mathrm{k}$-th power $\mathrm{M}_{\mathrm{m}} \# \ldots \# \mathrm{M}_{\mathrm{m}}$. Using the natural isomorphism

$$
s^{k}\left(M_{m}\right) \approx \frac{M_{m}^{k}}{M_{m}^{k-1}}
$$

we associate with the $k$-th order differential $\mathrm{d}^{\mathrm{k}} \varphi_{\mathrm{m}}: \mathrm{M}_{\mathrm{m}}^{\mathrm{k}} \rightarrow \mathrm{L}_{\varphi(\mathrm{m})}^{\mathrm{k}}$ the linear map

$$
\varphi_{\mathrm{m}}^{\mathrm{k}}: S^{\mathrm{k}}\left(\mathrm{M}_{\mathrm{m}}\right) \rightarrow \frac{\mathrm{L}^{\mathrm{k}}(\mathrm{m})}{\mathrm{d}^{\mathrm{k}-1} \varphi\left(\mathrm{M}_{\mathrm{m}}^{\mathrm{k}-1}\right)}
$$

To each $\mathrm{X}_{\mathrm{m}}$ we associate the linear subspaces of $\mathrm{N}_{\mathrm{m}}=\operatorname{ker} \mathrm{d} \varphi$ :

$$
\mathrm{N}_{1}(\mathrm{X}, \mathrm{m})=\left[\mathrm{A \varepsilon N} \mathrm{m}_{\mathrm{m}} ; \varphi_{\mathrm{m}}^{2}(\mathrm{X} \# \mathrm{~A})=0\right]
$$

and 


$$
N_{2}(X, m)=\left[A \varepsilon N_{1}(X, m) ; \varphi_{m}^{3}(X \# X \# A)=0\right]
$$

Let $\operatorname{ord}(\mathrm{m})=\operatorname{dim} \mathrm{N}_{\mathrm{m}}$ and $\mathrm{k}_{i}(\mathrm{X}, \mathrm{m})=\operatorname{dim} \mathrm{N}_{i}(\mathrm{X}, \mathrm{m})$ for $i=1,2$.

THEOREM. With the above notation if $\gamma$ is any smooth curve in $M$ tangent to $X$ at $m=\gamma(1)$ then there exists a real number $\varepsilon>0$ such that

$$
\text { ord } \gamma(\mathrm{t}) \leq \mathrm{k}_{2}(\mathrm{X}, \mathrm{m}) \leq \mathrm{K}_{1}(\mathrm{X}, \mathrm{m}) \leq \text { ord }(\mathrm{m})
$$

for all $t \neq 1,1-\varepsilon<t<1+\varepsilon$.

66.8 H.S.M. Coxeter (University of Toronto)

Mid-Circles and Loxodromes

Any two circles have at least one mid-circle which interchanges them by inversion. Two tangent or nonintersecting circles have a unique mid-circle, whose construction presents an interesting problem. Another surprisingly neglected subject is the family of loxodromes, which are inverses of equiangular spirals. These plane curves (related to the better known spherical loxodromes by stereographic projection) give their name to the most general kind of Möbius transformation: the "loxodromic" homography. It is interesting to see which centres of inversion will yield centrally symmetric loxodromes. A third problem is the construction of the invariant points of the Möbius involution $\left(A_{1} B_{1}\right)\left(A_{2} B_{2}\right)$ that interchanges two given pairs of points.

\subsection{K.K. Oberai (Queen's University) Spectrum of a Spectral Operator}

Foquel [Pac. J. Math. 8(1958), 51-65] proved that if $T$ is a spectral operator on a Banach space $E$ then the spectrum of $T$ coincides with its approximate spectrum. We give a simple example to show that this is not necessarily so if $E$ is a Frechet space and we prove: Let $E$ 
be a complete locally convex space and let $T$ be a spectral operator of finite type on $E$ with compact spectrum. Then the spectrum of $T$ coincides with its approximate spectrum (this result is actually proved with a slightly less restrictive condition on the spectrum of $T$ ).

$66.10 \mathrm{R}$. Iltis (University of Toronto)

Some Algebraic Structure in the Dual of a Compact Group

Let $G$ be a compact topological group and let $G^{-}$denote the set of equivalence classes of continuous, irreducible, unitary representations of $G$. An element $\gamma$ in $G^{-}$is called compact if the tensor powers of $\gamma$ and its conjugate contain only a finite number of elements of $\mathrm{G}^{-}$. The compact elements are precisely the members of $G^{-}$which annihilate (map onto an identity operator) the component of the identity in $G$. An element $\gamma$ in $G^{-}$is compact if and only if the range of $\gamma$ is finite. It follows that: (1) $G$ is connected if and only if the range of $\gamma$ is finite for all nontrivial $\gamma$ in $G^{-}$, and (2) $G$ is totally disconnected if and only if the range of $\gamma$ is finite for all $\gamma$ in $G^{-}$. Two applications of the preceeding results are: (1) If $G$ is periodic, then $G$ is totally disconnected, and (2) $G$ is connected if and only if $G$ is divisible.

\subsection{E. Zakon (University of Windsor)}

On the Essential Metrization of Uniform Spaces

Two uniformities (filters of "entourages") $\underset{\sim}{\mathrm{U}}$ and $\underset{\sim}{\mathrm{V}}$ on a space $T$ are said to be $m$-equivalent ( $m$ being an outer measure on all subsets of $T$ ) if, for any $U \varepsilon U_{\sim}$ and $V \varepsilon \underset{\sim}{V}$, there are $V_{0} \varepsilon \underset{\sim}{V}$ and $U_{0} \varepsilon \underset{\sim}{U}$ such that $\mathrm{m}\left(\mathrm{U}_{0}[\mathrm{x}]-\mathrm{V}[\mathrm{x}]\right)=0=\mathrm{m}\left(\mathrm{V}_{0}[\mathrm{x}]-\mathrm{U}[\mathrm{x}]\right)$ for all $\mathrm{x} \varepsilon \mathrm{T}$. We say that a uniform space $(T, \underset{\sim}{U})$ is essentially (pseudo) metrizable, with respect to $m$, if $(T, V)$ is (pseudo) metrizable in the ordinary sense, for some $\underset{\sim}{V}$ that is m-equivalent to $\underset{\sim}{U}$. Results: 
(1) A method is developed which makes it possible to construct in any uniform space $(T, \underset{\sim}{U})$ an outer measure $m$ (in general, not unique) under which $(T, \underset{2}{U})$ is essentially pseudometrizable.

(2) Several theorems are proved on essential pseudometrizability with respect to a prescribed $\mathrm{m}$. For example, a separable space $(T, \underset{\sim}{U})$ is essentially pseudometrizable with respect to $m$, if $\underset{\sim}{U}$ is $m$-equivalent to a uniformity $\underset{\sim}{V}$ with a nested base. Other such conditions apply to uniform Lindelöf spaces and to compact spaces.

\subsection{N. Dinculeanu (Queen's University) and C. Foiaş (University of Bucharest)} Algebraic Models for Measure Preserving Transformations

$(\Gamma, U, \varphi)$ is an algebraic ergodic system (a.e.s.) if $\Gamma$ is an abelian group, $U$ is an automorphism of $\Gamma$ and $\varphi$ is a function of positive type such that $\varphi \cdot U=\varphi$. Example: $T$ an invertible measure preserving (i.m.p.) transformation on a probability measure space $(X, \Sigma, \mu), \Gamma(\mu)$ the set of the equivalence classes $f \varepsilon L^{\infty}(\mu)$ with $|f| \equiv 1$, $U_{T} f=f \cdot T$ for $f \varepsilon L^{2}(\mu), \varphi_{\mu}(f)=\int f d \mu ;$ then $\left(\Gamma(\mu), U_{T}, \varphi_{\mu}\right)$ is an a.e.s.. An a.e.s. $(\Gamma, U, \varphi)$ is an algebraic model for $T$ if there exists an injective homomorphism $j: \Gamma \rightarrow \Gamma(\mu)$ with $U_{T^{j}}=j U$ and $\varphi=\varphi_{\mu} \cdot j$, such that $j \Gamma$ generates $L^{2}(\mu)$. Every $T$ has an algebraic model. Every a.e.s. is an algebraic model for some $T$. Conjugacy can be characterized by means of algebraic models. An a.e.s. $(\Gamma, U, \varphi)$ is discrete if $\Gamma$ contains the circle group $C$ and $\varphi(\gamma)=\gamma \varphi_{C}(\gamma)$ for $\gamma \in \Gamma$. The i.m.p. transformations with discrete algebraic models can be characterized by means of the $(\Gamma, U)$ part of algebraic models $(\Gamma, U, \varphi)$. These transformations contain the ergodic transformations with discrete spectrum and those with quasi-discrete spectrum. 